\title{
TYPE 1 KNOT INVARIANTS IN 3-MANIFOLDS
}

\author{
Paul Kirk and Charles Livingston
}

The general theory of knotting in 3-manifolds has recently seen significant progress. One important aspect of this has been the effort toward generalizing the notion of finite type invariants from $S^{3}$ to arbitrary 3-manifolds. Here we will present a new class of finite type invariants, defined in arbitrary orientable 3-manifolds, that are both simple to define and to compute. They will be seen to be of both practical utility, in distinguishing large families of knots, and also of theoretical interest, giving access to subtle unknotting results.

\section{Introduction.}

For knots in $S^{3}$ the theory of finite type invariants now offers a key perspective for the study of knot invariants. Excellent references for this background material include $[\mathbf{B a}, \mathbf{B}, \mathbf{B L}]$. In this setting the first nontrivial finite type invariant is the second coefficient of the Conway polynomial, which is also the second derivative of the Jones polynomial at 1 , and this is the only type 2 invariant. It is also known that for any fixed type, $n$, there is a finite bound on the rank of the space of type $n$ integral invariants; work of Kontsevich shows that those bounds are realized. (See $[\mathbf{B a}]$ for details.)

While the definition of finite type invariants extends directly to manifolds other than $S^{3}$ (see $[\mathbf{L i}, \mathbf{S}]$ ), this setting is far less understood. In [ $\left.\mathbf{L i}\right]$ it is shown that finite type invariants arising from polynomial invariants in $S^{3}$ extend to give invariants in homotopy spheres. Work of Stanford [S] concerning finite type invariants of links in $S^{3}$ can be viewed as offering insights into the study of embedded knots in classical knot and link exteriors. Of particular note with regards to extending the theory of finite type invariants to the more general setting is the work of Kalfagianni $[\mathbf{K a}]$, in which a beautiful interplay between classical 3-manifold techniques and more modern methods came to the fore. In $[\mathbf{K a}]$ it is proved that finite type invariants from $S^{3}$ can induce finite type invariants in irreducible 3-manifolds. It is also noted that, at least for irreducible rational homology spheres, the Conway polynomial offers access to other finite type invariants.

With the exceptions just mentioned, few explicit examples of finite type invariants in arbitrary 3-manifolds are known. Our work here constructs 
large new families of type 1 invariants. Our main goals are to describe these invariants, to prove that they are in fact well defined, and to examine their basic properties and applications. However, the results and approach are best appreciated in reference to those of Kalfagianni. Key insights that $[\mathbf{K a}]$ provided include: 1) In studying finite type invariants in a 3-manifold, one can focus separately on each conjugacy class in the fundamental group, 2) it is often immaterial that an invariant is defined in terms of a choice of basepoint, in this case the choice of a representative of a conjugacy class; the usefulness of an invariant in distinguishing knots is based on the fact that the difference of its values on two knots is well defined, independent of the choice of basepoint, and 3) the techniques of 3-manifold topology, in particular the theory of incompressible tori developed by Jaco-Shalen [JS] and Johannson [Jo], plays a central role in understanding finite type invariants. In a manner similar to that of $[\mathbf{K a}]$, the properties of injective tori in irreducible 3-manifolds will be crucial to understanding these new invariants. (Four-manifold techniques are used in the present work as well.) An additional observation is that the invariants developed here can be used to show that the results of $[\mathbf{K a}]$ do not generalize to knots in reducible 3manifolds (see Section 5G); more generally, these new invariants illustrate the central role played by the structure of the 3 -manifold in defining finite type knot invariants in arbitrary 3 -manifolds.

We now outline the contents of this paper. In Section 2 we set up notation and define the concept of a type $n$ invariant for a knot in a 3-manifold $M$. We show how a type 1 invariant is determined modulo type 0 invariants by its values on singular knots with one crossing. (In the 3 -sphere there are no nontrivial type 1 invariants, but we show in this paper that such exist in other 3-manifolds.) Section 3 carries out the construction of new type 1 knot invariants for knots in 3-manifolds. The construction starts with the choice of a 3-manifold, $M$, a free homotopy class, $\gamma \in\left[S^{1}, M\right]$, a nonnegative integer, $n$, and a cohomology class $\tau \in H^{1}(M ; \mathbf{Z} / n)$ which vanishes on $\gamma$. (Here $\mathbf{Z} / n$ denotes the cyclic group of order $n$ if $n$ is nonzero, and $\mathbf{Z}$ if $n=0$.) These invariants are defined for singular knots with exactly one double point; we then give intersection conditions on $M, \gamma$ and $\tau$ that imply that the invariant is induced by a type 1 knot invariant. These invariants take values in the free abelian group $\mathbf{Z}[\mathbf{Z} / n]$, the integral group ring of $\mathbf{Z} / n$. (This group is defined in detail in Section 3; note here that it is a torsion free group, isomorphic to $\mathbf{Z}^{n}$ if $n \neq 0$ and $\oplus_{i=1}^{\infty} \mathbf{Z}$ if $n=0$. Hence, the invariant can be viewed as a collection of $n$ integral type 1 invariants if $n \neq 0$ and an infinite collection of invariants if $n=0$. The independence of these invariants is addressed in Section 5.) An example demonstrating the ease of calculation is also given in Section 3. 
Section 4 is devoted to describing situations in which the intersection conditions imply that we have a well defined type 1 knot invariant. For example, we show that this invariant is well defined in all the following situations: 1) If $M$ is essentially any orientable irreducible 3-manifold that does not contain an $S^{1}$ bundles over a nonorientable surface; 2) if $M$ is obtained by surgery on a knot in a homology sphere and $n$ is a prime power; 3 ) if $\gamma$ is of finite order and $M$ is arbitrary. We also explore the extension of the invariant over connected sums of manifolds.

In Section 5 we present a number of observations and applications. First we give a realization result for the invariant and use this to find lower bounds on the rank of the vector space of $F$-valued type 1 invariants on a given free homotopy class for a field $F$. We also use the realization result to show that the group of integral type 1 invariants of nullhomotopic knots in a manifold with non-trivial integral first cohomology is uncountable. The next observation of the section is a relationship of the new invariant to the Conway polynomial; if the 3-manifold $M$ is the exterior of a knot $K$ in $S^{3}$ and the cohomology class $\tau$ represents the generator of $H^{1}(M ; \mathbf{Z} / n)$, then the composite of our invariant with a certain homomorphism $\mathbf{Z}[\mathbf{Z} / n] \longrightarrow \mathbf{Z} / n$ evaluated on a knot $J$ equals, modulo $n$, the third coefficient of the Conway polynomial of the link $(K, J)$.

Next in Section 5 we review our previous work [KL] on finite type link invariants in $S^{3}$ and contrast the two approaches. In particular we show that not all type 1 invariants of knots in a given homotopy class in $M$ are homomorphic images of one of the invariants defined in this paper. Following this we discuss the relationship of our invariants to the Kojima-Yamasaki $\eta$-function for two component links of linking number zero, which in some special cases gives a way to relate type 1 invariants for homologous but not homotopic knots.

Section 5 then presents a provocative result concerning unknotting operations. We show that if $\mathrm{K}$ represents a nontrivial homology class in an orientable 3-manifold, its positive double (which is obviously null homotopic) cannot be unknotted by making only positive crossing changes. Related results for knots in $S^{3}$ have been attained by Cochran and Lickorish [CL], but only by using the powerful tools of 4-manifold theory developed by Donaldson.

We conclude Section 5 with an example in $S^{1} \times S^{2}$ for which our invariant is not well defined. This in turn shows that general results of Kalfagianni that hold for (most) irreducible 3-manifolds cannot be extended to the reducible setting. In another paper, [KL2], we explore the issue of counterexamples fully, providing a converse to our main result here, via a complete description of the class of manifolds for which our invariant is well defined. 
We thank the referee for many valuable comments.

\section{Preliminaries.}

We begin by recalling what finite type invariants are. Here, and throughout this paper, we will assume all 3-manifolds are compact and oriented. Given such a 3 -manifold $M$, a knot invariant with values in an abelian group $A$ is a function

$$
v: \operatorname{Emb}\left(S^{1}, M\right) \longrightarrow A
$$

whose value depends only on the isotopy class of an embedding. Any invariant extends to the space of "nice" singular knots $\mathcal{S}(M)$ consisting of smooth immersions with double points that are transverse (in the sense that the two tangent vectors are independent) and no other singularities. The extension is given by the inductive definition

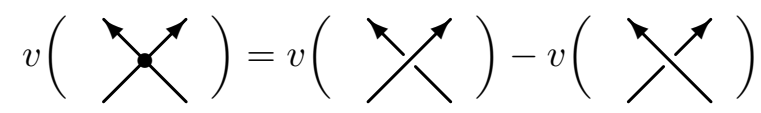

An invariant is called a Vassiliev invariant of type $n$ if its extension to $\mathcal{S}(M)$ vanishes whenever a singular knot has $n+1$ or more crossings. Note that a type $n$ invariant is also a type $n+k$ invariant for $k \geq 0$. Good references for details of the theory of finite type invariants include [Ba, B, BL, Li, S].

Type 0 Invariants. The invariants we define in Section 3 are all of type 1 , and we will want to consider them as elements of the group of type 1 invariants modulo the subgroup of type 0 invariants. Hence we need to begin with a few simple observations regarding type 0 invariants.

A type 0 Vassiliev invariant takes the same value on two knots that differ by a single crossing change. Thus the value of a type 0 invariant on a knot $K$ in $M$ depends only on the free homotopy class of $K$. It follows that, given an abelian group $A$, a type 0 invariant with values in $A$ is simply a function

$$
\left[S^{1}, M\right] \longrightarrow A,
$$

where $\left[S^{1}, M\right]$ denotes the set of free homotopy classes of maps from $S^{1}$ to $M$. If $M$ is simply connected, then the set $\left[S^{1}, M\right]$ consists of a single point and so the type 0 invariants are identified with $A$ itself. In general, the set of free homotopy classes in $M$ is in one-to-one correspondence with the set of path components of $\mathcal{S}(M), \pi_{0}(\mathcal{S}(M))$.

Type 1 Invariants. Next consider type 1 invariants. We begin by noting that in the 3 -sphere any type 1 invariant vanishes on all knots with one 
double point; that is, every type 1 invariant in $S^{3}$ is also of type 0 . To see this, let $v$ be a type 1 invariant. If $K$ is an immersion of $S^{1}$ into $S^{3}$ with one double point, then $v(K)=v\left(K^{\prime}\right)$ where $K^{\prime}$ is any immersion obtained from $\mathrm{K}$ by a single crossing change; this is because $v$ vanishes on knots with two double points. Since $S^{3}$ is simply connected, a sequence of crossing changes converts $K$ into a simple (planar) figure eight. The value of $v$ on the figure eight is 0 , since resolving the crossing point in either direction results in the same knot, the unknot. Hence $v(K)=0$ also.

Any type 1 invariant can be altered by adding to it a type 0 invariant. We will consider two type 1 invariants to be equivalent if they differ by a type 0 invariant; this is done formally by considering the invariant as a representative in the quotient of the group of type 1 invariants modulo the subgroup of type 0 invariants.

To put this another way, we are interested only in the difference of the value of an invariant on two freely homotopic knots, not in its value on any given knot. In this way, we view type 1 invariants as secondary invariants, and a type 1 invariant is determined modulo type 0 invariants by its value on the subspace of $\mathcal{S}(M)$ consisting of those singular knots with exactly one crossing. Given a free homotopy class $\gamma$ and a fixed embedded knot $K_{0}$ in $\gamma$, replacing a type 1 invariant $v$ by the invariant $v^{\prime}$ defined by $v^{\prime}(K)=$ $v(K)-v\left(K_{0}\right)$ yields an equivalent type 1 invariant which vanishes on $K_{0}$.

As already remarked in $[\mathbf{K a}]$, finite type invariants are best studied one path component of $\mathcal{S}(M)$ at a time. Thus we decompose $\mathcal{S}(M)$ into its path components

$$
\mathcal{S}(M)=\cup_{\gamma \in\left[S^{1}, M\right]} \mathcal{S}(M)_{\gamma},
$$

where $\mathcal{S}(M)_{\gamma}$ denotes the path component of $\mathcal{S}(M)$ containing a representative of $\gamma$. Given a path component $\mathcal{S}(M)_{\gamma}$, we will construct a type 1 invariant $v_{\tau}$ on $\mathcal{S}(M)_{\gamma}$ given any cohomology class $\tau \in H^{1}(M ; \mathbf{Z} / n)$ which vanishes on $\gamma$.

Definition 2.1. If $K_{0}$ and $K_{1}$ are homotopic embedded knots, a transverse homotopy from $K_{0}$ to $K_{1}$ is a homotopy $K_{t}, t \in[0,1]$, so that for $t \notin\left\{t_{0}, t_{1}, \cdots, t_{n}\right\}, K_{t}$ is an embedding, and $K_{t_{i}}$ is a singular knot with one transverse crossing such that $K_{t_{i}+\epsilon}$ and $K_{t_{i}-\epsilon}$ are the two non-singular resolutions of $K_{t_{i}}$ (in some order) for small enough $\epsilon$. (A transverse homotopy always exists between any two homotopic knots.)

Fix $\gamma \in\left[S^{1}, M\right]$, and fix a knot $K_{0}: S^{1} \subset M$ in the free homotopy class $\gamma$. Suppose that $v: \mathcal{S}(M)_{\gamma} \longrightarrow A$ is a type 1 invariant and $K_{1}$ is a knot homotopic to $K_{0}$. The difference $v\left(K_{1}\right)-v\left(K_{0}\right)$ is the signed sum of the values of $v$ on the singular knots $K_{t_{i}}$ for a transverse homotopy $K_{t}$. (It is useful for what follows to observe that the sign is also the local oriented 
self-intersection number of the 2-dimensional trace of the homotopy in the 4-manifold $M \times\left[t_{i}-\epsilon, t_{i}+\epsilon\right]$.)

In summary:

Proposition 2.2. A type 1 invariant is determined by:

1. Its value on some chosen (embedded) knot $K_{\gamma}$ in the free homotopy class $\gamma$ for each $\gamma \in\left[S^{1}, M\right]$, and,

2 . its value on the subspace of $\mathcal{S}(M)$ consisting of those singular knots with exactly one crossing.

Modulo type 0 invariants a type 1 invariant is determined by the second condition alone.

We are interested in constructing type 1 invariants. Denote by $\Sigma^{1} \subset \mathcal{S}(M)$ the subspace of knots with one crossing. Its intersection with $\mathcal{S}(M)_{\gamma}$ will be denoted by $\Sigma_{\gamma}^{1}$. Given a singular knot $K: S^{1} \longrightarrow M$ in $\Sigma_{\gamma}^{1}$, we call the two oriented loops in the image of $K$ the lobes of $K$.

Proposition 2.2 implies that a type 1 invariant $v: \mathcal{S}(M)_{\gamma} \longrightarrow A$ is determined modulo type 0 invariants by its values on $\Sigma_{\gamma}^{1}$. In other words, the restriction of a type 1 invariant $v$ to $\Sigma_{\gamma}^{1}$ gives a function $f: \Sigma_{\gamma}^{1} \longrightarrow A$ which in turn determines $v$ up to type 0 invariants.

Thus to construct a type 1 invariant we must find a function on $f: \Sigma_{\gamma}^{1} \longrightarrow A$ 

Section 4 describes settings for which $v_{\tau}$ is a well defined type 1 knot invariant, including most irreducible 3-manifolds, all hyperbolic 3-manifolds, all Seifert fibered manifolds over orientable surfaces of positive genus, and all irreducible rational homology spheres. Applications to reducible manifolds will also be given. To prepare the groundwork for those results, we will next develop a simple intersection condition that implies that $v_{\tau}$ is well defined. First though we offer a simple example to illustrate how easy $v_{\tau}$ is to compute. The example occurs in the solid torus, (for which it will be seen that $v_{\tau}$ is well defined) but note that by results of Section 4 , most embeddings of the solid torus into irreducible 3-manifolds that are injective on homology can be used to construct further examples in fairly arbitrary irreducible 3-manifolds. In Section 5 we will provide many more examples that exist in far more general classes of 3-manifolds.

Example. Let $M$ be the solid torus. Then $\left[S^{1}, M\right]=\pi_{1} M=\mathbf{Z}$. Let $\gamma_{n}$ denote the free homotopy class which represents $n$ times the generator. Each singular knot in $\Sigma_{\gamma_{n}}^{1}$ determines an unordered pair of integers $k, n-k$, namely the winding numbers of the two lobes of $K$. Let $\tau \in H^{1}(M ; \mathbf{Z} / n)$ denote the generator. The invariant $v_{\tau}: \mathcal{S}(M)_{\gamma} \longrightarrow A(n)$ is determined by the property that it takes a singular knot in $\Sigma_{\gamma}^{1}$ to $t^{k}+t^{n-k}-2$.
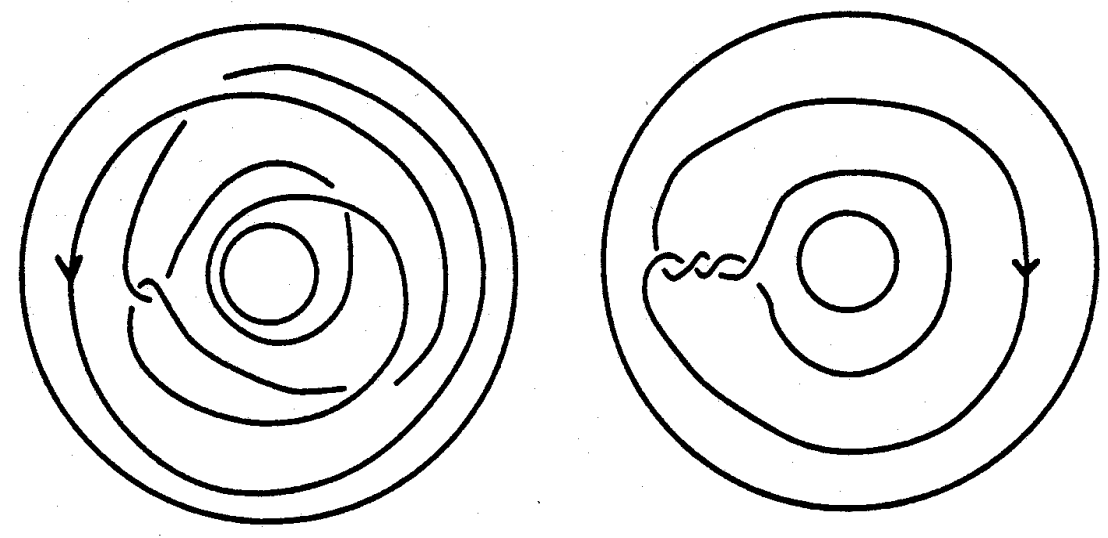

Figure 1.

Thus to compute the difference of the value of $v_{\tau}$ on two knots $K_{0}$ and $K_{1}$ in the solid torus which wind $n$ times around, one takes a transverse homotopy of $K_{0}$ to $K_{1}$. Then for each parameter $t_{i}$ corresponding to a singular knot with one crossing, the value of $v_{\tau}$ changes by the product of the sign of the double point and the quantity $t^{k}+t^{-k}-2$ for the appropriate $k$. Figure 1 shows a pair of nullhomotopic knots in the solid torus whose 
invariants differ by $2\left(t+t^{-1}-2\right)+t^{2}+t^{-2}-2$. (One can take a band sum of these examples with a fixed winding number $n$ knot to obtain examples with winding number $n$.)

The Intersection Condition. As we explained in Section 2, the function $f_{\tau}: \Sigma_{\gamma}^{1} \longrightarrow A$ defines an invariant $V_{\tau}\left(K_{t}\right)$ of transverse homotopies $K_{t}$, and this in turn well-defines a type 1 invariant $v_{\tau}\left(K_{1}\right)$ provided that $V_{\tau}$ vanishes on any homotopy from a knot to itself. Here we reinterpret the vanishing of $V_{\tau}\left(K_{t}\right)$ in terms of equivariant intersection numbers.

Still working with a fixed $M, \gamma$, and $\tau$, let $\Gamma$ be a map of a torus $S^{1} \times S^{1}$, into $M$ which restricts to $\gamma$ on $S^{1} \times *$, and suppose that $\tau$ pulls back to be the trivial class on the torus via $\Gamma$. Let $\tilde{M}$ denote the cover of $M$ induced by $\tau$, let $\alpha \in H_{2}(\tilde{M}, \mathbf{Z})$ be the homology class represented by a lift of $\Gamma$ to $\tilde{M}$, and let $\alpha_{0} \in H_{1}(\tilde{M}, \mathbf{Z})$ be the homology class represented by the corresponding lift of $\gamma$ to $\tilde{M}$. With this notation we have the following theorem.

Theorem 3.1 (Intersection Condition). $v_{\tau}$ is a well defined type 1 knot invariant if and only if, for all $\Gamma$ as above,

$$
\sum_{s=0}^{n-1}\left(\alpha \cdot\left(t^{s}+t^{-s}\right) \alpha_{0}\right)\left(t^{s}-1\right)=0
$$

in $\mathbf{Z}[\mathbf{Z} / n]$, where the sum is infinite if $n=0$.

The rest of this section gives the proof of this result.

Four-manifold methods. Fix a singular knot with one double point, $K_{0}$. Using $K_{0}$ construct a transverse homotopy $K_{t}$ with one crossing from the positive resolution $K_{+}$to the negative resolution $K_{-}$of $K_{0}$. The trace of this homotopy is a level preserving immersion

$$
k: S^{1} \times I \longrightarrow M \times I
$$

with one double point so that

$$
k(-, t)= \begin{cases}K_{+} & \text {if } t<1 / 2 \\ K_{0} & \text { if } t=1 / 2 \\ K_{-} & \text {if } t>1 / 2\end{cases}
$$

Fix orientation conventions so that this double point has sign +1 in the oriented 4-manifold $M \times I$.

The homomorphism $\tau: H_{1} M \longrightarrow \mathbf{Z} / n$ defines the $\mathbf{Z} / n$ cover

$$
\tilde{M} \longrightarrow M
$$


and hence a cover

$$
\tilde{M} \times I \longrightarrow M \times I .
$$

Moreover, the homotopy $k: S^{1} \times I \longrightarrow M \times I$ lifts to $\tilde{k}: S^{1} \times I \longrightarrow \tilde{M} \times I$ (since $\left.\left[k\left(S^{1} \times\{t\}\right)\right]=\gamma\right)$.

The equivariant intersection form

$$
\langle,\rangle: H_{2}(\tilde{M} \times I, \partial(\tilde{M} \times I)) \times H_{2}(\tilde{M} \times I, \partial(\tilde{M} \times I)) \longrightarrow \mathbf{Z}[\mathbf{Z} / n]
$$

is defined as usual by

$$
\langle z, w\rangle=\sum_{t^{s} \in \mathbf{Z} / n}\left(z \cdot t^{-s} w\right) t^{s}
$$

There is also the projection to the augmentation ideal

$$
p: \mathbf{Z}[\mathbf{Z} / n] \longrightarrow \mathbf{Z}[\mathbf{Z} / n]
$$

defined by $p\left(t^{s}\right)=t^{s}-1$.

A simple argument shows that

$$
V_{\tau}\left(K_{t}\right)=p(\langle\tilde{k}, \tilde{k}\rangle)
$$

By composing transverse homotopies one then obtains the following lemma:

Lemma 3.2. Let $K_{0}, K_{1}$ be homotopic embedded knots in $M$ representing $\gamma$. Choose a transverse homotopy $K_{t}$ from $K_{0}$ to $K_{1}$ and let $\tilde{K}_{t}: S^{1} \times I \longrightarrow \tilde{M}$ be a lift of the homotopy to the $\mathbf{Z} / n$ cover. Denote by $\tilde{k}: S^{1} \times I \longrightarrow \tilde{M} \times I$ the trace of $\tilde{K}_{t}$. Then

$$
V_{\tau}\left(K_{t}\right)=p(\langle\tilde{k}, \tilde{k}\rangle)
$$

From this lemma we conclude that $v_{\tau}$ is a well defined type 1 invariant on $\mathcal{S}(M)_{\gamma}$ if and only if $p(\langle\tilde{k}, \tilde{k}\rangle)=0$ whenever $\tilde{k}$ is constructed from a self-homotopy of $K_{0}$. In this reformulation one is no longer bound to the crossing change formula, since any homology (and in particular homotopy) of $\tilde{k}$ will give the same value of $\langle\tilde{k}, \tilde{k}\rangle$.

The case of $\mathbf{n} \neq \mathbf{0}$. We will first complete the proof of the intersection condition when $n \neq 0$, in which case the cover $\tilde{M} \longrightarrow M$ is a finite cyclic cover, and then derive the case $n=0$ as a consequence.

Thus assume that $n \neq 0$. We are given a homotopy $K_{t}$ from an embedded knot $K_{0}$ to itself, and we must show that $p(\langle\tilde{k}, \tilde{k}\rangle)=0$ is implied by the intersection condition, where $\tilde{k}$ denotes the trace of the lift of the homotopy to the $n$-fold cover $\tilde{M} \longrightarrow M$. 
First notice that if we perform the homotopy $m$ times in succession, obtaining the lift $\tilde{k}^{\prime}$ say, then

$$
p\left(\left\langle\tilde{k}^{\prime}, \tilde{k}^{\prime}\right\rangle\right)=m \cdot p(\langle\tilde{k}, \tilde{k}\rangle)
$$

In particular, $p\left(\left\langle\tilde{k}^{\prime}, \tilde{k}^{\prime}\right\rangle\right)$ vanishes if and only if $p(\langle\tilde{k}, \tilde{k}\rangle)$ does. By choosing $m$ appropriately (e.g. $m=n$ ) we can therefore arrange that the lift $\tilde{K}_{t}$ is also a self-homotopy.

Hence we can shift our point of view slightly and consider the homotopy $K_{t}$ as giving a map $k: S^{1} \times S^{1} \longrightarrow M \times S^{1}$ and a lift of this map $\tilde{k}: S^{1} \times$ $S^{1} \longrightarrow \tilde{M} \times S^{1}$. This is more convenient since what must be shown from this perspective is that the equivariant intersection $p(\langle\tilde{k}, \tilde{k}\rangle)$ of the absolute 2-dimensional homology class $[\tilde{k}]$ in $\tilde{M} \times S^{1}$ equals zero.

Next we use the Kunneth formula to relate intersection numbers in $M \times S^{1}$ to intersection numbers in $M$. Suppose that $K_{t}: S^{1} \longrightarrow M, t \in[0,1]$ denotes the self-homotopy and $\tilde{K}_{t}: S^{1} \longrightarrow \tilde{M}, t \in[0,1]$ its lift. View $\tilde{K}_{t}$ as a map of a torus into $\tilde{M}$, representing a class which we denote by $\alpha \in H_{2}(\tilde{M})$. Also, let $\alpha_{0} \in H_{1}(\tilde{M})$ be the class represented by $\tilde{K}_{0}: S^{1} \longrightarrow \tilde{M}$. Then the homology class $\tilde{k}$ in $\tilde{M} \times S^{1}$ equals the sum of $i_{*}(\alpha)$ and $i_{*}\left(\alpha_{0}\right) \times\left[S^{1}\right]$ where $i: \tilde{M} \longrightarrow \tilde{M} \times S^{1}$ denotes the inclusion $m \mapsto(m, 1)$.

Using the Kunneth theorem one sees:

$$
\begin{aligned}
\tilde{k} \cdot t^{-s} \tilde{k} & =i_{*}(\alpha) \cdot t^{-s}\left(i_{*}\left(\alpha_{0}\right) \times\left[S^{1}\right]\right)+\left(i_{*}\left(\alpha_{0}\right) \times\left[S^{1}\right]\right) \cdot t^{-s} i_{*}(\alpha) \\
& =\alpha \cdot\left(t^{s}+t^{-s}\right) \alpha_{0}
\end{aligned}
$$

where the last line denotes intersections in the 3 -manifold $\tilde{M}$.

Hence, summing we have that $p\left(\left\langle\tilde{k}^{\prime}, \tilde{k}^{\prime}\right\rangle\right)=0$ if

$$
\sum_{s=0}^{n-1}\left(\alpha \cdot\left(t^{s}+t^{-s}\right) \alpha_{0}\right)\left(t^{s}-1\right)=0
$$

in $\mathbf{Z}[\mathbf{Z} / n]$, as desired.

The case of $\mathbf{n}=\mathbf{0}$. The case $n=0$ can be reduced to the case $n \neq 0$ in the following way. The self-homotopy defines an intersection number $p(\langle\tilde{k}, \tilde{k}\rangle)$ in $\mathbf{Z}[\mathbf{Z}]=\mathbf{Z}\left[t, t^{-1}\right]$ which we wish to show vanishes. Choosing $n$ larger than the degree of $p(\langle\tilde{k}, \tilde{k}\rangle)$ we can work in the intermediate $\mathbf{Z} / n$ cover to conclude that the image of the Laurent polynomial $p(\langle\tilde{k}, \tilde{k}\rangle)$ in $\mathbf{Z}[t] /\left(t^{n}-1\right)$ is zero, using the lemma and the device of replacing the homotopy by its $n$-fold composite. Since the degree of $p(\langle\tilde{k}, \tilde{k}\rangle)$ is less than $n$, this implies that $p(\langle\tilde{k}, \tilde{k}\rangle)$ vanishes in $\mathbf{Z}[\mathbf{Z}]$. This concludes the proof. 


\section{Setting for which $v_{\tau}$ is a well defined knot invariant.}

We now want to describe situations in which the intersection condition can be applied to show that $v_{\tau}$ is a well defined knot invariant. Again, our initial data is the oriented manifold $M$, the free homotopy class $\gamma$ and the cohomology class $\tau \in H^{1}(M, \mathbf{Z} / n)$ that vanishes on $\gamma$. Our condition says that $v_{\tau}$ is well defined if for all tori $\Gamma: S^{1} \times S^{1} \rightarrow M$ for which $\Gamma\left(S^{1} \times *\right)=\gamma$ and such that the pullback of $\tau$ to $S^{1} \times S^{1}$ via $\Gamma$ is trivial we have

$$
\sum_{s=0}^{n-1}\left(\alpha \cdot\left(t^{s}+t^{-s}\right) \alpha_{0}\right)\left(t^{s}-1\right)=0
$$

where $\alpha$ and $\alpha_{0}$ are lifts of $\gamma$ and $\Gamma$ to the n-fold cover of $M, \tilde{M}$.

We begin with two elementary conditions on $\gamma$, and follow this with general conditions on $M$.

\section{Conditions on $\gamma$.}

Theorem 4.1. $\quad v_{\tau}$ is a well defined knot invariant if $\tilde{\gamma}$ is of finite order in $H_{1}(\tilde{M})$.

Proof. If $\tilde{\gamma}$ is of finite order in $H_{1}(\tilde{M})$ all intersections $t^{s} \alpha \cdot \alpha_{0}$ will certainly be 0 , since the intersection number takes values in $\mathbf{Z}$ which is torsion free.

Corollary 4.2. $\quad v_{\tau}$ is a well defined knot invariant if $\gamma$ has finite order in $\pi_{1}(M)$, or if $\gamma$ lies in the second commutator subgroup of $\pi_{1}(M)$.

Example. In Section 5 we will explore the case of $\gamma$ trivial, providing fascinating information regarding unknotting operations. So we present here an example with $\gamma$ essential. Let $J$ be an arbitrary knot in $S^{3}$, or in any homology sphere $\Sigma^{3}$. Let $M$ denote the space obtained by performing $p / q$ surgery on $J$, so that $H_{1}(M, Z)=\mathbf{Z} / p$. Suppose that $n$ is a prime power divisor of $p$. The n-fold cyclic cover of $M$ is a rational homology sphere, since it is constructed by $(p / n) / q$ surgery on the the lift of $J$ to the n-fold branched cover of $\Sigma^{3}$ over $J$. This lift is null homologous (it bounds the lift of a Seifert surface for $J$ ) and the prime power branched cover of $\Sigma^{3}$ is a rational homology sphere. Hence, if $\tau \in H^{1}(M, \mathbf{Z} / n)$ is a nontrivial class, then $v_{\tau}$ yields a well defined type 1 knot invariant for any homotopy class in the kernel of $\tau$. If $J$ is the unknot in $S^{3}$ we are considering a lens space. In most other cases there are an infinite number of such homotopy classes. Notice that $M$ need not be irreducible, even if we start with $S^{3}$. As the simplest example, $p q$ surgery on the $(p, q)$ torus knot yields the connected sum of lens spaces, $L(p, q) \# L(q, p)$. 
Conditions on $M$ : the set of admissible irreducible 3-manifolds, $\mathcal{N}$.

We prefer to have conditions on $M$ that hold for all possible $\gamma$ and $\tau$. While it is not quite true that irreducibility is enough ([KL2] presents counterexamples as well as necessary and sufficient conditions) by eliminating a few special 3-manifolds irreducibility becomes sufficient. Roughly, the orientable irreducible manifolds for which $v_{\tau}$ is not well defined are those manifolds containing circle bundles over non-orientable surfaces along with three particular closed orientable Seifert fibered manifolds which contain non-vertical tori.

For notation, let $M(F)$ denote the unique circle bundle over the punctured surface $F$ for which the total space is orientable. In [KL2] we show how the presence of such spaces in the case that $F$ is a punctured nonorientable surface leads to the failure of $v_{\tau}$ to be well defined.

There are seven orientable Seifert fibered 3-manifolds that contain incompressible tori which cannot be homotoped to be vertical. Four of these have base space $S^{2}$ : these are $M\left(S^{2},(2,1),(3,-1),(6,-1)\right) M\left(S^{2},(3,1),(3,1)\right.$, $(3,-2)) \quad M\left(S^{2},(2,1),(4,-1),(4,-1)\right) \quad$ and $\quad M\left(S^{2},(2,1),(2,1),(2,-1)\right.$, $(2,-1))$. (Here, for instance, $M\left(S^{2},(2,1),(3,-1),(6,-1)\right)$ is the unique Seifert fibered space over $S^{2}$ with three singular fibers and Seifert invariants $(2,1),(3,-1)$, and $(6,-1)$.) One has base space $P^{2}$ and two singular fibers, $M\left(P^{2},(2,1),(2,1)\right)$, and the last two are the $S^{1}$ bundles over the torus and Klein bottle with total space orientable.

Of these seven, $M\left(S^{2},(2,1),(2,1),(2,-1),(2,-1)\right)$ is homeomorphic to the circle bundle over the Klein bottle, and hence contains a circle bundle over a nonorientable surface, as does $M\left(\mathbf{R} P^{2},(2,1),(2,1)\right)$. Also, every injective map of a torus into $T^{3}$ is vertical with respect to some Seifert fibration. We abbreviate the remaining three Seifert fiber spaces by $M_{(2,3,6)}$, $M_{(2,4,4)}$, and $M_{(3,3,3)}$.

Definition. Let $\mathcal{N}$ denote the set of all orientable irreducible 3-manifolds excluding any that contain $M(F)$ for $F$ nonorientable, and also excluding the three manifolds $M_{(3,3,3)}, M_{(2,4,4)}$, and $M_{(2,3,6)}$.

These constraints are quite limited; for instance, easy exercises in 3manifold theory give the following.

Theorem 4.3. The set $\mathcal{N}$ contains:

(1) All irreducible $\mathbf{Z} / 2$ homology spheres,

(2) all orientable hyperbolic 3-manifolds,

(3) all Seifert fibered spaces over orientable surfaces of positive genus, 
(4) all but four Seifert fibered spaces with base space $S^{2}$, and

(5) all bounded 3-manifolds with $H_{2}(M, \mathbf{Z} / 2)=0$, for instance knot complements in homology spheres.

One can find other manifolds in $\mathcal{N}$, for example any irreducible rational homology sphere $M$ with $H_{1}(M ; \mathbf{Z})=\mathbf{Z} / 2 \oplus T$ for $T$ an odd torsion abelian group is in $\mathcal{N}$.

The main result of this section is the next theorem. In [KL2] we will prove a converse which essentially states that $v_{\tau}$ is a well defined knot invariant for all $\tau$ if and only if $M$ is the connected sum of a manifold in $\mathcal{N}$ and a homology sphere. (The only needed modification to this statement is that certain $S^{1}$ bundles over punctured Möbius bands need not be excluded.)

Theorem 4.4. If $M$ is in $\mathcal{N}$, then $v_{\tau}$ is a well defined knot invariant.

This result is a consequence of the following lemma. (Notice that if $n=0$, $v_{\tau}$ is well defined if $v_{\tau_{n}}$ is well-defined for sufficiently large $n$, where $\tau_{n}$ denotes the reduction of $\tau$ modulo $n$.)

Lemma 4.5. Let $\tilde{M} \longrightarrow M$ be any $n$-fold cyclic cover of a 3-manifold $M$ in $\mathcal{N}$. Let $\alpha: S^{1} \times S^{1} \longrightarrow \tilde{M}$ be any map of a torus into $\tilde{M}$, and let $\alpha_{0}: S^{1} \longrightarrow \tilde{M}$ denote the restriction of $\alpha$ to the first factor. Then

$$
\sum_{s=0}^{n-1}\left(\alpha \cdot\left(t^{s}+t^{-s}\right) \alpha_{0}\right)\left(t^{s}-1\right)=0
$$

in $\mathbf{Z}[\mathbf{Z} / n]=\mathbf{Z}[t] /\left(t^{n}-1\right)$.

Remark. Our proof of this lemma uses the Jaco-Shalen-Johannson theorem and is inspired by Kalfagianni's use of this theorem in $[\mathbf{K a}]$. For basic results concerning 3-manifolds, see Hempel [He].

Proof of Lemma 4.5. If $\pi_{1} M$ is finite, then as in Theorem 4.1, $\alpha_{0}$ is finite order, so all intersections with $\alpha_{0}$ are trivial. Thus we assume that $\pi_{1} M$ is infinite. This implies that $M$ is aspherical and $\tilde{M}$ is irreducible [He].

If the map $\alpha: T^{2} \longrightarrow \tilde{M}$ is not injective on fundamental groups, then since $\pi_{1}(\tilde{M})$ is torsion free, $\alpha$ can be compressed, but then $[\alpha] \in H_{2}(\tilde{M})$ is represented by a spherical class, which must be trivial since $\pi_{2} \tilde{M}=0$. Thus the intersection number of $\alpha$ with $t^{s} \alpha_{0}$ vanishes for any $s$.

If the map $\alpha: T^{2} \longrightarrow \tilde{M}$ is homotopic into the boundary, the intersection number of $\alpha$ with $t^{s} \alpha_{0}$ vanishes for any $s$ because $t^{s} \alpha_{0}$ can be homotoped to be disjoint from $\alpha$. 
This leaves the case when $\alpha: T^{2} \longrightarrow \tilde{M}$ is an essential torus. Let $\beta$ : $T^{2} \longrightarrow M$ denote the composite of $\alpha$ with the covering projection $\tilde{M} \longrightarrow M$. Then $\beta$ is an essential map. If $M$ is Haken, then by the Jaco-ShalenJohannson theorem [JS, Jo], $\beta$ is homotopic into the characteristic submanifold of $M$, and in fact must be homotopic into a Seifert fibered component $C$ of the characteristic submanifold, since the only orientable twisted $I$-bundle containing a $\mathbf{Z} \oplus \mathbf{Z}$ in its fundamental group is the twisted $I$-bundle over the Klein bottle, which is Seifert fibered over the Möbius band. If $M$ is not Haken, then the theorem of Gabai $[\mathbf{G a}]$ and Casson-Jungreis [CJ] implies that $M$ is Seifert fibered. In this latter case let $C=M$.

Let $\tilde{C}$ denote the inverse image of $C$ in $\tilde{M}$. It is also Seifert fibered, being a finite cover of $C$. A homotopy of $\beta$ into $C$ lifts to give a homotopy of $\alpha$ into $\tilde{C}$.

We can conclude that $\beta$ is homotopic to a vertical torus in $C$, since by restricting to $\mathcal{N}$ we have eliminated all Seifert fibered spaces with nonvertical tori. The homotopy lifts to a homotopy of $\alpha$ in $\tilde{C}$ and does not change the intersection numbers.

Let $C_{0} \longrightarrow F_{0}$ denote the restriction of the Seifert fibration of $C$ to the complement of a neighborhood of the singular fibers. Similarly define $\tilde{C}_{0} \longrightarrow \tilde{F}_{0}$. Note that since $M$ is in $\mathcal{N}, F_{0}$ is orientable. The torus $\alpha\left(T^{2}\right)$ is vertical, and by a small homotopy if necessary we may assume that there is an immersed curve $\gamma$ in $F_{0}$ so that $\alpha\left(T^{2}\right)$ is the union of the regular fibers over $\gamma$.

The circle bundle $C_{0} \longrightarrow F_{0}$ is trivial and so we identify $F_{0}$ with a section of this bundle. Similarly the circle bundle $\tilde{C}_{0} \longrightarrow \tilde{F}_{0}$ is trivial; use the pull back of the section of $C_{0} \longrightarrow F_{0}$ to trivialize it. Thus one has a fiber preserving $\mathbf{Z} / n$ action on the bundle $\tilde{C}_{0} \longrightarrow \tilde{F}_{0}$ with quotient $C_{0} \longrightarrow F_{0}$.

Since $\alpha\left(T^{2}\right)$ is vertical, the intersection number of $\alpha$ with any fiber in the fibration $\tilde{C}_{0} \longrightarrow \tilde{F}_{0}$ is zero. Therefore, by subtracting a multiple of the fiber from $\alpha_{0}$ we may assume that $\alpha_{0}$ lies in the base $\tilde{F}_{0}$ of the fibration (or more properly a section).

We have reduced the lemma to the following simple situation. A trivial circle fibration $\tilde{C}_{0} \longrightarrow \tilde{F}_{0}$ with a $\mathbf{Z} / n$ action over a surface is given, with quotient a circle fibration $C_{0} \longrightarrow F_{0}$. An immersed curve $\alpha_{0}$ in $\tilde{F}_{0}$ is given, determining the immersed torus $\alpha$ in $\tilde{C}_{0}$ as the union of the fibers over $\alpha_{0}$. We must show that the intersection numbers $\alpha \cdot\left(t^{s}+t^{-s}\right) \alpha_{0}$ vanish.

Because $\tilde{F}_{0}$ is orientable, the intersection number $\alpha \cdot\left(t^{s}+t^{-s}\right) \alpha_{0}$ in $\tilde{C}_{0}$ equals the intersection number $\alpha_{0} \cdot\left(t^{s}+t^{-s}\right) \alpha_{0}$ in the $\mathbf{Z} / n$-surface $\tilde{F}_{0}$. Since $F_{0}$ is orientable, the restriction of the covering transformation $t^{s}$ to $\tilde{F}_{0}$ is orientation preserving, and so

$$
\alpha_{0} \cdot t^{s} \alpha_{0}=t^{-s} \alpha_{0} \cdot \alpha_{0}=-\alpha_{0} \cdot t^{-s} \alpha_{0}
$$


Thus $\alpha_{0} \cdot\left(t^{s}+t^{-s}\right) \alpha_{0}=0$.

This completes the proof of Lemma 4.5 and hence the intersection condition holds for $M \in \mathcal{N}$. Theorem 4.4 follows.

Theorem 4.4 says that for manifolds in $\mathcal{N}$, the invariant $v_{\tau}$ is well defined for all $\tau$ and $\gamma$. The collection of manifolds for which $v_{\tau}$ is well defined can be extended to include connected sums of manifolds in $\mathcal{N}$ with homology spheres, as the following theorem implies. (As mentioned earlier, in [KL2] we will prove a converse which essentially says that unless a manifold is a connected sum of a manifold in $\mathcal{N}$ and a homology sphere, there exists some $\tau$ so that $v_{\tau}$ is not well defined. The only modification needed to make this precise is that certain $S^{1}$ bundles over punctured Möbius bands need not always be omitted.)

Theorem 4.6. Suppose that for some irreducible manifold $M$ and $\tau \in$ $H^{1}(M ; \mathbf{Z} / n)$, the invariant $v_{\tau}$ is well defined for all homotopy classes $\gamma \in$ $\operatorname{ker} \tau$. Let $H$ be a homology sphere and let $\bar{\tau} \in H^{1}(M \# H ; \mathbf{Z} / n)$ denote the unique extension of $\tau$ to the connected sum. Then $v_{\bar{\tau}}$ is well defined for all homotopy classes $\gamma \in \operatorname{ker} \bar{\tau}$.

Proof. It suffices to assume that $n \neq 0$. Given a knot $K$ in $N=M \# H$ with $\bar{\tau}([K])=0$ and a self homotopy $K_{t}$ of $K$ which lifts to the n-fold cover $\tilde{N}$, we must check that the intersection condition holds. Think of the lift of the self-homotopy as a map of the torus into $\tilde{N}$.

Notice that $\tilde{N}$ is the connected sum of the $n$-fold cover $\tilde{M}$ of $M$ defined by $\tau$ and $n$ copies of $H$. Let $S \in \tilde{N}$ denote the preimage of the 2-sphere separating $M$ from $H$, so that $S$ is the union of $n 2$-spheres in $\tilde{N}$ separating $\tilde{M}$ from the copies of $H$.

Call the lift of the self-homotopy $\alpha: T^{2} \longrightarrow \tilde{N}$ and the lift of the knot $\alpha_{0}: S^{1} \subset T^{2} \longrightarrow \tilde{N}$. Make $\alpha$ transverse to $S$. The preimage of $S$ by $\alpha$ is then a collection of embedded circles in $T^{2}$.

If one of these circles is essential in $T^{2}$, then $\alpha$ can be compressed because $S$ is a union of embedded 2-spheres. Thus the homology class in $H_{2}(\tilde{N})$ represented by $\alpha: T^{2} \longrightarrow \tilde{N}$ is spherical. The Sphere Theorem (see [He]) implies either that this class is trivial or that $\tilde{N}$ is a connected sum of a manifold with $S^{1} \times S^{2}$. But $\tilde{N}$ cannot contain such a summand because its prime decomposition is unique and $\tilde{M}$ is irreducible. Thus the homology class $\alpha$ is trivial and hence the intersection numbers $t^{s} \alpha_{0} \cdot \alpha$ vanish. Thus in this case the intersection condition holds.

Suppose that instead every circle in the preimage of $S$ by $\alpha$ is inessential. Then the circle $S^{1} \subset T^{2}$ representing the lift of the knot $K$ can be isotoped 
off of these circles (in $T^{2}$ ), and hence the knot $K$ lies either entirely in $M$ or entirely in $H$. In the latter case its lift lies entirely in one of the copies of $H$ in $\tilde{N}$ and so it is nullhomologous and the intersection condition must hold.

This leaves the case when $\alpha_{0}\left(S^{1}\right)$ lies entirely in $\tilde{M}$ and $\alpha\left(T^{2}\right)$ meets the collection $S$ of 2 -spheres in inessential circles. In the standard way one can use the product structure near $S$ to ambiently surger $\alpha\left(T^{2}\right)$; This gives a representative of the homology class $[\alpha]$ consisting of one torus containing $\alpha_{0}$ which lies entirely in $\tilde{M}$ and some 2 -spheres each of which must represent the zero class by the argument of the previous paragraph. But now the expression $\left(t^{s}+t^{-s}\right)\left[\alpha_{0}\right] \cdot[\alpha]$ must vanish since $v_{\tau}$ is well-defined for $M$. This concludes the proof of Theorem 4.6.

Remark. Suppose that the image of $\tau: H_{1} M \longrightarrow \mathbf{Z} / n$ has index $k$. Write $n=m k$. Let $\tau^{\prime} \in H^{1}(M ; \mathbf{Z} / m)$ correspond to the surjection obtained restricting the range of $\tau$ to $\mathbf{Z} / m \subset \mathbf{Z} / n$. Then $v_{\tau}(t) \in \mathbf{Z}[\mathbf{Z} / n]$ is obtained from $v_{\tau^{\prime}}(t) \in \mathbf{Z}[\mathbf{Z} / m]$ by replacing the variable $t$ by $t^{k}$, since the corresponding cover has $k$ path components and we are counting intersections in one component only. Thus all the information contained in these invariants can be obtained by considering the primitive $\tau \in H^{1}(M ; \mathbf{Z} / n)$, that is, those $\tau$ which are surjective as homomorphisms $\pi_{1} M \longrightarrow \mathbf{Z} / n$.

Further examples. The methods we have just described can be combined, and used with other 3-manifold techniques to yield a vast array of results. We conclude this section with a sampling of possibilities.

Theorem 4.7. If $v_{\tau}$ is well defined on a manifold $M$, then for any 3manifold $N, v_{\tau^{\prime}}$ gives a well defined invariant on $M \# N$, where $\tau^{\prime}$ is the natural extension of $\tau$ and $v_{\tau}^{\prime}$ is defined for homotopy classes $\gamma$ that can be represented by curves in $M$.

Proof. Let $S$ be the 2-sphere yielding the connected sum decomposition, $M \# N=\left(M-B^{3}\right) \cup\left(N-B^{3}\right)$. Given a knot $K$ representing $\gamma$ in $M$, let $K_{t}$ be a self homotopy, the image of which is a torus, $T$. If $T$ misses $S$ we are done, so we assume $T$ intersects $N-B^{3}$ in a subsurface $F$ of $T$; notice that $F$ does not intersect $K$ since $K$ is in $M-B^{3}$. Replace the subsurface $F$ on $T$ with a homeomorphic surface, $F^{\prime}$, contained in a neighborhood of $S$. This will not affect the intersection condition, since neither the lifts of $F$ nor of $F^{\prime}$ intersect $K$. We now have a torus that is homotopic to one in $M-B^{3}$, so, since $v_{\tau}$ was well defined, the intersection condition must hold for this torus. Hence, for all tori the intersection condition holds, and it follows that $v_{\tau^{\prime}}$ is well defined.

Note that if $M \in \mathcal{N}$ and $N$ is a 3-dimensional submanifold of $M$, then $N$ is 
also in $\mathcal{N}$. Next we observe that under appropriate homological conditions, any $v_{\tau}$ defined on $N$ extends to give a well defined knot invariant on $M$. The proof is immediate. Notice that in the case that $N$ is a solid torus this result shows that the examples in the solid torus that we constructed in Section 3 can be used to generate examples in arbitrary manifolds $M$ in $\mathcal{N}$ as long as $H_{1}(M) \neq 0$.

Theorem 4.8. Let $N \subset M \in \mathcal{N}$ and suppose that $\tau \in H^{1}(N, \mathbf{Z} / n)$ is in the image of $H^{1}(M, \mathbf{Z} / n)$. Then the invariant $v_{\tau}$ defined on $N$ extends to $M$. In particular, if $H_{1}(N, \mathbf{Z} / n) \rightarrow H_{1}(M, \mathbf{Z} / n)$ is injective, then every such $v_{\tau}$ extends to $M$.

\section{Properties of the invariants.}

Here we present a series of results and observations concerning $v_{\tau}$. We assume throughout that we are in a setting in which $v_{\tau}$ is well defined - for instance, any of the cases described in Section 4.

A. Realization results. In Section 3 we showed how to construct type 1 invariants

$$
v_{\tau}: \mathcal{S}(M)_{\gamma} \longrightarrow \mathbf{Z}[\mathbf{Z} / n]
$$

from any $\tau \in H^{1}(M ; \mathbf{Z} / n)$ which vanishes on $\gamma$, for $M$ an irreducible, oriented 3-manifold. The invariant takes values in the subgroup $A(n)$ generated by the elements $t^{s}+t^{-s}-2$. It would be unfortunate if this invariant were always zero. We begin this section by showing that in fact the opposite is true: any element in $A(n)$ is $v_{\tau}$ on some knot $K_{1}$, provided $\tau$ is primitive. (Note that if $n$ is prime or $\tau$ generates an infinite summand of $H_{1}(M ; \mathbf{Z})$ then it is automatically primitive.) We use this to find lower bounds on the rank of the space of type 1 integral invariants in special cases. In particular we show that if $\gamma$ is a homotopy class on which some integral cohomology class $\tau \in H^{1}(M ; \mathbf{Z})$ vanishes, then the group of integral (or $F$-valued for any field $F$ ) type 1 invariants is uncountable.

Theorem 5.1. Let $\tau \in H^{1}(M, \mathbf{Z} / n)$ be a primitive class. Let $K_{0} \subset M$ be any (embedded) knot with $\tau\left(\left[K_{0}\right]\right)=0$. Then given any $p(t) \in A(n)$, there exists an embedded knot $K_{1}$ homotopic to $K_{0}$ with

$$
v_{\tau}\left(K_{1}\right)-v_{\tau}\left(K_{0}\right)=p(t) .
$$

Proof. It suffices by induction to prove this theorem in the case when $p(t)=$ $\pm\left(t^{s}+t^{-s}-2\right)$. Let $\alpha$ be any loop based at some point of $K_{0}$ with $\tau(\alpha)=s$. 
Such an $\alpha$ exists given that we are working with a primitive class. Construct a homotopy of $K_{0}$ to a knot $K_{1}$ by pushing a "finger" of $K_{0}$ along $\alpha$ and then introducing a positive or negative clasp.

It follows from the crossing change formula that for the resulting knot $K_{1}$,

$$
v_{\tau}\left(K_{1}\right)=v_{\tau}\left(K_{0}\right)+ \pm\left(t^{s}+t^{-s}-2\right),
$$

the sign depending on whether the clasp is taken positive or negative.

Remark. If $\tau$ is not primitive a similar argument shows that if $\tau=k \phi$ for some primitive class $\phi$, then $v_{\tau}$ is onto the subgroup of $A(n)$ generated by the elements $t^{k s}+t^{-k s}-2$.

One can take homomorphic images of the abelian groups $A(n)$ to get other (weaker) invariants. This is quite easy since $A(n)$ is a free abelian group. If $A$ is any abelian group, one can map $t^{s}+t^{-s}-2$ into $A$ arbitrarily for each $s$. This defines an $A$-valued type 1 Vassiliev invariant

$$
v_{\tau}: \mathcal{S}(M)_{\gamma} \longrightarrow A
$$

for any choice of values for $t^{s}+t^{-s}-2$. Generalizing this idea we obtain the following.

Corollary 5.2. Let $\gamma \in\left[S^{1}, M\right]$. Suppose that for some $n$ the kernel of the homomorphism $H^{1}(M ; \mathbf{Z} / n) \longrightarrow \mathbf{Z} / n$ given by evaluating a cohomology class on $\gamma$ is non-zero. If $F$ is a field, the vector space of $F$-valued type 1 modulo type 0 invariants on $\mathcal{S}(M)_{\gamma}$ has dimension at least $\left[\frac{n}{2}\right]$ if $n \neq 0$ and is infinite dimensional (and uncountable) if $n=0$. Similarly the group of integer-valued invariants on $\mathcal{S}(M)_{\gamma}$ contains a free abelian subgroup of rank at least $\left[\frac{n}{2}\right]$ if $n \neq 0$ and is uncountable if $n=0$.

Proof. Let $\operatorname{Emb}\left(S^{1}, M\right)_{\gamma}$ denote the set of embedded knots that are homotopic to $\gamma$. Let $F$ denote either a field or $\mathbf{Z}$. Fix a knot $K_{0}$ in the homotopy class $\gamma$. Choose a primitive $\tau$ in the kernel of the evaluation $e_{\gamma}: H^{1}(M ; \mathbf{Z} / n) \longrightarrow \mathbf{Z} / n$, given by evaluating a cohomology class on $\gamma$. Then the invariant $v_{\tau}: \operatorname{Emb}\left(S^{1}, M\right)_{\gamma} \longrightarrow A(n)$ determined by $\tau$ and the condition that $v_{\tau}\left(K_{0}\right)=0$ is onto by the previous theorem. It follows that the induced homomorphism

$$
\operatorname{Hom}(A(n), F) \longrightarrow \operatorname{Map}\left(E m b\left(S^{1}, M\right)_{\gamma}, F\right)
$$

is injective and has image contained in the space of type 1 invariants.

Since $A(n)$ has rank $\left[\frac{n}{2}\right]$ if $n \neq 0, \operatorname{Hom}(A(n), F)$ has rank $\left[\frac{n}{2}\right]$. On the other hand, if $n=0, A(0)$ is free abelian of infinite rank, and so $\operatorname{Hom}(A(0), F)$ is infinitely generated and uncountable. 
One can get better bounds than those of this corollary by considering the size of the first homology group.

Theorem 5.1 applies to provide a large variety of examples; the following corollary captures many such cases.

Corollary 5.3. Let $M$ be an orientable 3-manifold with nontrivial first homology. Then there is a type 1 invariant on $M$ that distinguishes an infinite family of knots. If $M \in \mathcal{N}$ has noncyclic first homology then every homotopy class in $M$ contains an infinite collection of distinct knots distinguished by $v_{\tau}$ for the appropriate choice of $\tau$.

Proof. The first statement is simple to prove. $H^{1}(M ; \mathbf{Z} / n)$ is nontrivial for some $n$. Pick such an $n$ and a primitive class $\tau \in H^{1}(M ; \mathbf{Z} / n)$. Let $U$ denote the unknot. Now Theorem 5.1 applies to construct a homotopic knot $K_{1}$ with

$$
v_{\tau}\left(K_{1}\right)-v_{\tau}(U)=p(t),
$$

where $p(t)$ can be selected arbitrarily in $A(n)$. Since $A(n)$ is infinite, the result follows.

For the second statement, pick an embedded representative $K_{0}$ of the desired homotopy class. Then there is always a choice of $n$ and $\tau$ so that the conditions of Theorem 5.1 are satisfied. To see this, pick a prime $n$ for which $H_{1}(M ; \mathbf{Z} / n)$ is not cyclic. Then $H^{1}(M ; \mathbf{Z} / n)$ is at least 2-dimensional, so we can find a class $\tau$ that vanishes on any fixed class in $H_{1}(M ; \mathbf{Z} / n)$. Now Theorem 5.1 applies to construct a homotopic knot $K_{1}$ with

$$
v_{\tau}\left(K_{1}\right)-v_{\tau}\left(K_{0}\right)=p(t),
$$

where $p(t)$ can be selected arbitrarily in $A(n)$. Again, since $A(n)$ is infinite, the result follows.

Remark. The set of such manifolds is extensive. For instance, by $[\mathrm{M}]$ every finitely generated abelian group occurs as the first homology for some oriented hyperbolic closed 3-manifold. Other examples follow from the fact that all Seifert fibered spaces that fiber over a surface of positive genus also satisfy the hypothesis [He].

B. The Conway Polynomial. Let $K$ be a knot in the complement of a second knot $L$ in $S^{3}$. Here we relate our invariants to the value of the third coefficient $C_{3}$ of the Conway polynomial of the link $(L, K)$.

We first remind the reader of the (easily proven) fact that if $(L, K(+))$ and $(L, K(-))$ are two 2-component links in $S^{3}$ with the same first component and whose second component differ by a single crossing change,

$$
C_{3}((L, K(+)))-C_{3}((L, K(-)))=a b+b c+c a
$$


where $a, b, c$ denote the three linking numbers of the 3 -component link obtained by smoothing the crossing.

Now let $L \subset S^{3}$ be a knot and let $M$ denote the complement of a neighborhood of $L$. (Recall that Theorem 4.3 implies that knot complements are in $\mathcal{N}$.) Let $\tau \in H^{1}(M, \mathbf{Z})=\mathbf{Z}$ denote the generator. Suppose that $K(+), K(-)$ are knots in $M$ which differ by a single crossing change and for which $\tau(K( \pm))=n$, i.e. the linking number of $L$ and $K( \pm)=n$. Denote by $A, B$ the two components of the smoothed intermediate singular knot. Then

$$
v_{\tau}(K(+))-v_{\tau}(K(-))=t^{s}+t^{-s}-2 \in \mathbf{Z}[\mathbf{Z} / n]
$$

where $s$ denotes the linking number of $K$ with $A$ (or $B$ ). Letting $c$ denote the linking number of $A$ with $B$ from the previous paragraph one sees that

$$
C_{3}(L, K(+))-C_{3}(L, K(-))=s(n-s)+c s+c(n-s)=s(n-s)+c n .
$$

This immediately implies:

Theorem 5.4. Let $L \subset S^{3}$ be an (embedded) knot, and let $K_{i}: S^{1} \subset$ $S^{3}-L, i=0,1$ be two homotopic knots so that the linking number of $L$ with $K_{i}$ equals $n$. Let $\tau \in H^{1}\left(S^{3}-L ; \mathbf{Z}\right)=\mathbf{Z}$ be a generator (so that $\tau\left(K_{i}\right)=n$ ). Let $v_{\tau}: \mathcal{S}\left(S^{3}-L\right)_{\left[K_{0}\right]} \longrightarrow A(n)$ denote the type 1 invariant associated to $\tau$.

Then

$$
\Phi\left(v_{\tau}\left(K_{1}\right)-v_{\tau}\left(K_{0}\right)\right)=C_{3}\left(L, K_{1}\right)-C_{3}\left(L, K_{0}\right) \quad(\bmod n)
$$

where $\Phi: A(n) \longrightarrow \mathbf{Z} / n$ is the homomorphism taking $t^{s}+t^{-s}-2$ to $-s^{2}$.

Remark. One can construct knots $K_{0}$ and $K_{1}$ in $S^{3}-L$ so that $v_{\tau}\left(K_{0}\right)-$ $v_{\tau}\left(K_{1}\right)=0$ but $C_{3}\left(L, K_{0}\right)-C_{3}\left(L, K_{1}\right)$ is any multiple of $n$. This is done by adjusting the number $c$ in the discussion preceding this theorem by introducing crossing changes between the two lobes of a singular knot and letting $K_{0}$ and $K_{1}$ be its two resolutions.

Remark. Recall that as an invariant of links in $S^{3}, C_{3}$ is type 3. A relatively easy argument, using (5.1), shows that $C_{3}$ is type 2 when viewed as an invariant of knots in the complement of some fixed knot in $S^{3}$. It only becomes type 1 when restricted to knots that link the fixed knot $n$ times and when reduced modulo $n$.

C. A related invariant in homology circles. In $[\mathrm{KL}]$ we constructed an integral invariant $\lambda$ of 2-component links in $S^{3}$. Fixing one of the components, we can view $\lambda$ as an invariant of knots in classical knot complements. 
In fact, $\lambda$ generalizes to give a simple integer invariant of knots in homology circles. The definition of $\lambda$ mimics that of $v$, except for the final formula. We briefly recall the definition.

Let $\tau \in H^{1}(M ; \mathbf{Z})$ be cohomology generator, viewed as a homomorphism

$$
\tau: H_{1}(M ; \mathbf{Z}) \longrightarrow \mathbf{Z} .
$$

Also, fix a homotopy class $\gamma$ and let $n=\tau(\gamma)$. Use $\tau$ to define a function $g_{\tau}$ : $\Sigma_{\gamma}^{1} \longrightarrow \mathbf{Z}$ as follows. If $K: S^{1} \longrightarrow M$ is a singular knot with one double point, as before, let $\alpha$ and $\beta$ denote the two lobes of $K$, viewed as 1-dimensional homology classes. Now, define:

$$
g_{\tau}(K)=\tau(\alpha) \tau(\beta) .
$$

Finally, use Formulas (2.1) and (2.2) to define $\lambda$. The results of $[\mathbf{K L}]$ show that the resulting invariant $\lambda$ is well-defined.

Despite the similarity in their definitions, one can easily construct pairs of knots in $S^{1} \times D^{2}$ for which one or the other of $\lambda$ or $v_{\tau}$ distinguishes the knots but the other does not.

In addition to their similarity of definition, $\lambda$ is also related to the Conway polynomial, as is $v_{\tau}$ according to the preceding discussion; one has that $\lambda\left(K_{1}\right)-\lambda\left(K_{0}\right)$ reduces modulo $n$ to $C_{3}\left(L, K_{1}\right)-C_{3}\left(L, K_{0}\right)$ since $s(n-s)$ is congruent to $-s^{2}$ modulo $n$.

The invariant $\lambda$ can be used to show that not all type 1 invariants are composites of the $v_{\tau}$ and homomorphisms $A(n) \longrightarrow A$. To see this, consider $M=S^{1} \times D^{2}$, and choose the homotopy class $\gamma$ to represent the generator. Then only the zero cohomology class vanishes on $\gamma$, and so our construction yields only the trivial invariant. However, $\lambda: \mathcal{S}(M)_{\gamma} \longrightarrow \mathbf{Z}$ is non-trivial; its image is $2 \mathbf{Z}([\mathbf{K L}])$. Thus $\lambda$ cannot be the homomorphic image of some $v_{\tau}$.

In the next subsection we show that similar statements hold for any nontrivial $\gamma$ in $\left[S^{1}, S^{1} \times D^{2}\right]$. This example also indicates that the failure of the $v_{\tau}$ to detect all type 1 (integral) invariants occurs even in a manifold with an abelian fundamental group.

D. Knots in the solid torus. Let $M=S^{1} \times D^{2}$. Let $\gamma$ denote a free homotopy class. Identifying $H_{1} M$ with the integers identifies $\gamma$ with the integer $n$, say. Each singular knot $K$ in $\Sigma_{\gamma}^{1}$ defines a pair of integers $k, n-k$, namely the winding number of the two lobes of $K$.

Consider first $n=0$. Thus one is looking at type 1 invariants of nullhomotopic knots in the solid torus. We have the following result.

Theorem 5.5. Let $A$ be any abelian group. The group of type 1 invariants of nullhomotopic knots in the solid torus with values in $A$ (modulo type 0 
invariants) is isomorphic to the the group of functions $f: \mathbf{Z} \longrightarrow A$ which satisfy $f(0)=0$ and $f(-k)=f(k)$. The correspondence takes an invariant $v$ to the function $f(k)=v(S(k))$, where $S(k)$ is any singular knot with one crossing in the solid torus with winding number 0 such that one of the two lobes of $S(k)$ has winding number $k$.

Proof. Let $\tau \in H^{1}\left(S^{1} \times D^{2}, \mathbf{Z}\right)$ denote the generator. Since $\gamma$ is nullhomotopic, $\tau(\gamma)=0$. Then $\tau$ defines the type 1 invariant $v_{\tau}$ with values in the free abelian group $A(0)$. Note that $A(0)$ is freely generated by the polynomials $t^{k}+t^{-k}-2$ for $k \in \mathbf{Z}$.

Suppose that $f: \mathbf{Z} \longrightarrow A$ is any function satisfying $f(0)=0$ and $f(-k)=$ $f(k)$. Define a homomorphism $F: A(0) \longrightarrow A$ by sending $t^{k}+t^{-k}-2$ to $f(k)$. The composite of $v_{\tau}$ and $F$ is a type 1 invariant which takes the value $f(k)$ on any singular knot with one double point such that one of the lobes has winding number $k$.

Conversely, given any type 1 invariant, define $f(k)$ by $f(k)=v(S(k))$ where $S(k)$ is any nullhomotopic singular knot with one crossing one of whose lobes has winding number $k$. Then it is easy to check that $f(k)$ satisfies the two conditions $f(0)=0$ and $f(-k)=k$.

As an example, the coefficient of $t^{s}+t^{-s}-2$ in $v_{\tau}$ corresponds to the function $f$ with $f(s)=f(-s)=1$ and $f(k)=0$ if $k$ is not equal to $s$ or $-s$. The corresponding integral invariant is the algebraic count of the number of "type $s$ " crossings needed to unknot the knot. (Here we have chosen the trivial knot as a base point.)

Now consider the cases $n \neq 0$. If $\gamma$ has winding number $n$, then the same proof shows that any function $f: \mathbf{Z} \longrightarrow A$ which satisfies $f(0)=0$, $f(k)=f(n-k)$, and in addition the extra condition $f(k)=f(n+k)$ comes from a well-defined type 1 invariant. Just replace $A(0)$ by $A(n)$ in the above proof. But the argument also shows that if $v: \mathcal{S}_{\gamma} \longrightarrow A$ is a type 1 invariant which comes from a homomorphic image of $v_{\tau}$ (it suffices to take $\tau$ to be the generator of $\left.H^{1}\left(S^{1} \times D^{2} ; \mathbf{Z} / n\right)\right)$ then the associated function $f$ satisfies $f(k)=f(n+k)$.

However, the invariant $\lambda$ described in the previous subsection satisfies $\lambda(S(k))=k(n-k)$. Therefore, $\lambda(S(k))-\lambda(S(n+k))=2 k n \neq 0$ and hence $\lambda$ does not come from any $v_{\tau}$.

In [A] a type 1 invariant of knots in $S^{1} \times D^{2}$ is constructed, valid for all winding numbers. This invariant does reduce to $\lambda$, but its definition does not extend to homology circles, or even to classical knot complements.

\section{E. Relationship to Kojima and Yamasaki's $\eta$-function.}

In $[\mathrm{KoY}]$, Kojima and Yamasaki define an invariant of 2-component link- 
ing number zero links in $S^{3}$ with values in $\mathbf{Q}(t)$ which is closely related to our invariant. Given a link $(K, L)$ in $S^{3}$ with linking number zero, let $X$ denote the infinite cyclic cover of $S^{3}-L$. The inclusion $K \subset S^{3}-L$ lifts to $\tilde{K} \subset X$, and since $H^{1}(X ; \mathbf{Z})$ is torsion over $\mathbf{Z}[\mathbf{Z}], f(t) \tilde{K}$ bounds a 2-chain $\xi$ in $X$ for some polynomial $f(t)$. Then

$$
\eta(K, L)=\frac{1}{f(t)} \sum_{i=-\infty}^{\infty}\left(\xi \cdot t^{i} \tilde{K}\right) t^{i}
$$

(The constant term is fixed by lifting the 0-framing of $K$.)

Suppose that $K_{0}$ and $K_{1}$ are homotopic nullhomologous knots in $S^{3}-L$. The homotopy lifts to the infinite cyclic cover of $S^{3}-L$, and so one can choose the 2-chain $\xi_{1}$ to be the sum of $\xi_{0}$ and $f(t)$ times the lift of the homotopy. It follows that the difference $\eta\left(K_{1}, L\right)-\eta\left(K_{0}, L\right)$ is a Laurent polynomial and equals the equivariant self-intersection number of the lift of the homotopy, that is,

$$
\eta\left(K_{1}, L\right)-\eta\left(K_{0}, L\right)=v_{\tau}\left(K_{0}\right)\left(t^{-1}\right)-v_{\tau}\left(K_{1}\right)\left(t^{-1}\right)
$$

where $\tau \in H^{1}\left(S^{3}-L ; \mathbf{Z}\right)$ denotes the generator. Notice that if $K$ is nullhomotopic in $S^{3}-L$ then $\eta(K, L)$ is a Laurent polynomial and $\eta(K, L)(t)=$ $v_{\tau}(K)\left(t^{-1}\right)$, with $v_{\tau}$ normalized to vanish on a split link.

The $\eta$ invariant makes sense for any nullhomologous knot in a $\mathbf{Z}$-homology knot complement. Hence the difference $\eta\left(K_{1}\right)-\eta\left(K_{0}\right)$ is a well-defined rational function for any pair of nullhomologous knots $K_{0}$ and $K_{1}$ in a homology knot complement, and hence one can "define" $v_{\tau}\left(K_{1}\right)-v_{\tau}\left(K_{0}\right)$ for nullhomologous but not necessarily homotopic knots using Equation 5.2. This gives an intriguing way to relate type 1 invariants corresponding to homologous but not homotopic knots.

One faces difficulty in extending these ideas to the general $v_{\tau}$ invariants. For example, the first homology of an infinite cyclic cover of a 3-manifold is not usually torsion over $\mathbf{Z}[\mathbf{Z}]$.

\section{F. Unknotting operations and doubled knots.}

If $K$ is a null homotopic knot (in an arbitrary oriented 3-manifold) it can be unknotted by a series of crossing changes. However, crossing changes can be classified as either positive or negative, depending on whether the crossing change switches a negative crossing to a positive crossing or conversely. Determining what type of crossing changes are required to unknot a null homotopic knot is a very subtle problem. For instance, Cochran and Gompf [CG] were able to prove that the (positive) untwisted double of the trefoil knot in $S^{3}$ cannot be unknotted using only positive crossing changes; their argument depends critically on Donaldson's results concerning 4-manifolds. 
In contrast to these difficulties that occur in $S^{3}$, we have the following results.

Theorem 5.6. If $K$ represents a nontrivial homology class in an orientable 3-manifold, then the positive double, $D(K)$ of $K$ cannot be unknotted using only positive crossing changes.

Proof. $D(K)$ is null homotopic, so Theorem 4.1 applies to show that for every $\tau, v_{\tau}$ is well defined. Pick a cohomology class $\tau$, with coefficients in $\mathbf{Z} / n$ for some $n$, for which $v_{\tau}(K)=s \neq 0$. Then $v_{\tau}(D(K))-v_{\tau}$ (unknot) $=$ $t^{-s}-2+t^{s}$. Positive crossing changes applied to a knot $J$ either decrease the constant term of $v_{\tau}(J)$ or leave it unchanged. Hence, the -2 constant term in $v_{\tau}(D(K))-v_{\tau}$ (unknot) cannot be eliminated using only positive crossing changes.

Remark. By paying attention to all the coefficients of the polynomial and by using different homology classes, somewhat more technical arguments provide far stronger unknotting results. For instance, if the order of the first homology is greater than 3, one can construct null homotopic knots that require $a$ positive crossing changes and $b$ negative crossing changes to unknot, where $a$ and $b$ can be chosen arbitrarily.

\section{G. Connected sums with $S^{1} \times S^{2}$.}

Suppose that $M$ is the connected sum of a manifold $M^{\prime}$ and $S^{1} \times S^{2}$. Thus one can find an embedded 2-sphere $\phi: S^{2} \subset M$ and an embedded circle $\alpha: S^{1} \longrightarrow M$ which intersect transversally in one point. Let $\tau \in H^{1} M$ denote the Poincaré dual of $[\phi] \in H_{2} M$, viewed as a homomorphism $\pi_{1} M \longrightarrow \mathbf{Z}$.

The solid torus drawn in Figure 2 represents a neighborhood of $\alpha$ in $M$, and knots $K_{0}$ and $K_{1}$ are illustrated. (In the figure, $n=4$.) The obvious crossing changes transform $K_{0}$ into $K_{1}$, showing that the difference of the value of $v_{\tau}$ on the two knots is

$$
v_{\tau}\left(K_{1}\right)-v_{\tau}\left(K_{0}\right)=\sum_{s=0}^{n-1}\left(t^{s}+t^{-s}-2\right) \neq 0 .
$$

However, one can use the embedded sphere transverse to $\alpha$ to show that $K_{0}$ and $K_{1}$ are isotopic. Since the summation is not in general 0 , it follows that $v_{\tau}$ is not well defined. 

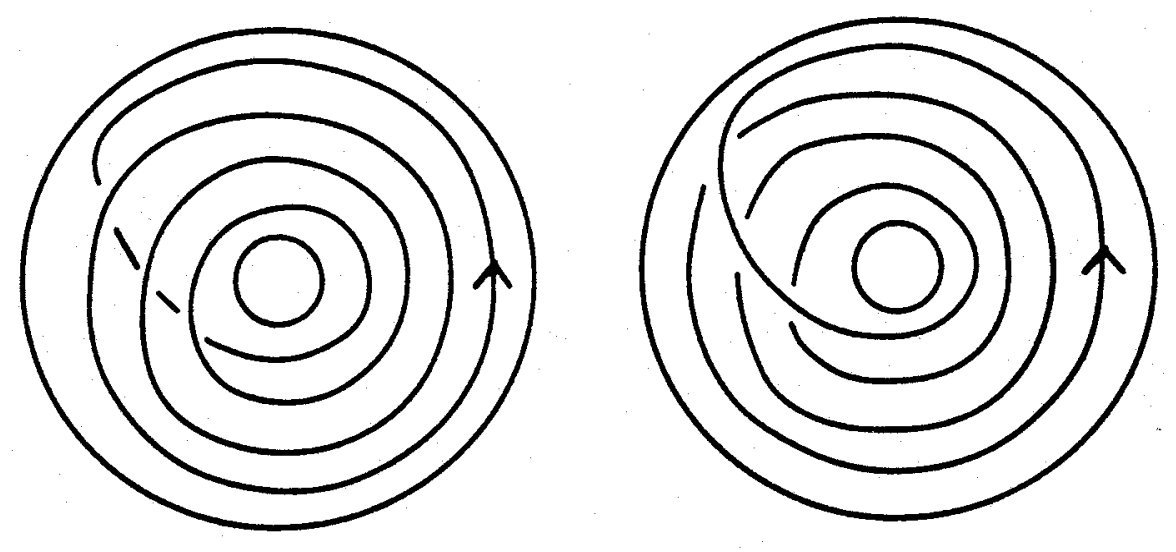

Figure 2.

We conjecture that the corresponding invariant in the quotient $A(n) / \sum\left(t^{s}+t^{-s}-2\right)$ is well defined.

Note that this construction illustrates the necessity of irreducibility in this paper and in work such as that of Kalfagianni. In [KL2] we take up the construction of examples that illustrate the necessity of the other restrictions that we have placed on our manifolds.

\section{References}

[A] F. Aicardi, Topological invariants of knots and framed knots in the solid torus, C. R. Acad. Sci. Paris Sr. I Math., 321 (1995), 81-86.

[Ba] D. Bar-Natan, On the Vassiliev knot invariants, Topology, 34 (1995), 423-472.

[B] J. Birman, New points of view in knot theory, Bulletin of the A.M.S., 28(2) (1993), 253-287.

[BL] J. Birman and X-S. Lin, Knot polynomials and Vassiliev's invariants, Invent. Math., 111 (1993), 225-270.

[CJ] A. Casson and D. Jungreis, Convergence groups and Seifert fibered 3-manifolds, Invent. Math., 118 (1994), 441-456.

[CG] T. Cochran and R. Gompf, Applications of Donaldson's theorems to classical knot concordance, homology 3-spheres and property P, Topology, 27 (1988) 495-512.

[CL] T. Cochran and W.B.R. Lickorish, Unknotting information from 4-manifolds, Trans. Amer. Math. Soc., 297 (1986), 125-142.

[G] D. Gabai, Convergence groups are Fuchsian groups, Ann. of Math., 136(2) (1992), 447-510.

[He] J. Hempel, 3-manifolds, Annals of Math. Studies Number 86, Princeton University Press, Princeton, New Jersey, 1976.

[JS] W. Jaco and P. Shalen, Seifert-fibered spaces in 3-manifolds, Memoirs of the A.M.S., 21(220) (1978). 
[Jo] K. Johannson, Homotopy equivalences of 3-manifolds with boundaries, Springer LNM Vol 761, Springer Verlag.

[Ka] E. Kalfagianni, Finite type invariants for knots in 3-manifolds, preprint, 1995.

[KL] P. Kirk and C. Livingston, Vassiliev invariants of 2-component links and the CassonWalker invariant, Topology, to appear.

[KL2] Knot invariants in 3-manifolds, essential tori, and the first cohomology of the free loop space on $M$, preprint, 1997.

[KoY] S. Kojima and M. Yamasaki, Some new invariants of links, Inventiones Math., 54 (1979), 213-228.

[Li] X-S. Lin, Finite type link invariants of 3-manifolds, Topology, 33 (1994), 45-71.

[M] R. Myers, Homology cobordisms, link concordances, and hyperbolic 3-manifolds, Trans. Amer. Math. Soc., 278 (1983), 271-288.

[S] T. Stanford, The functoriality of Vassiliev-type invariants of links, braids, and knotted graphs, J. Knot Theory Ramifications, 3 (1994), 247-262.

Received January 16, 1996 and revised March 27, 1997.

INDIANA UNIVERSITY

BLOOMINGTON, IN 47405

E-mail address: pkirk@ucs.indiana.edu

E-mail address: livingst@ucs.indiana.edu

NOTE: AN INCORRECT REFERENCE THAT APPEARED IN THE PAPER VERSION WAS REMOVED FROM THE FOURTH FULL PARAGRAPH ON PAGE 326. 